\title{
Original
}

Article

\section{Non-invasive Evaluation of Internal Thoracic Artery Anastomosed to the Left Anterior Descending Artery with 320-Detector Row Computed Tomography and Adenosine Thallium-201 Myocardial Perfusion Scintigraphy}

\author{
Yasushi Takagi, MD, Kiyotoshi Akita, MD, Hiroshi Kondo, MD, Michiko Ishida, MD, \\ Kan Kaneko, MD, Masato Sato, MD, and Motomi Ando, MD
}

\begin{abstract}
Purpose: We evaluated the relationship between internal thoracic artery (ITA) stenosis anastomosed to the left anterior descending artery (LAD) and the degree of LAD stenosis using 320-detector row computed tomography (320-ADCT) and adenosine thallium-201 myocardial perfusion scintigraphy (Tl-201-MPS).

Methods: We included 101 patients who underwent coronary artery bypass grafting (CABG) using ITA grafts; 320-ADCT and adenosine Tl-201-MPS were performed 2-3 months after CABG. Clinical parameters, degree of LAD stenosis, and regional myocardial ischemia of the LAD territory were compared between patients without ITA stenosis (Group A) and with ITA stenosis (Group B).

Results: Thirty patients $(30 \%)$ had $\leq 75 \%$ LAD stenosis, and 9 patients $(30 \%)$ showed significant ITA stenosis. Regional ischemia was noted in 23 patients $(23 \%)$. There were no differences in clinical parameters between the 2 groups. Twenty-two patients (24\%) in Group A and 8 patients $(89 \%)$ in Group B had $\leq 75 \%$ LAD stenosis $(\mathrm{P}<\mathbf{0 . 0 0 2})$. No Group B patients had regional myocardial ischemia of the LAD territory.

Conclusion: We concluded that $\leq 75 \%$ LAD stenosis significantly influences ITA stenosis, without associated regional myocardial ischemia of the LAD territory. Non-invasive 320ADCT and adenosine Tl-201-MPS for ITA evaluation may be useful for long-term follow-up of patients after CABG.
\end{abstract}

Keywords: internal thoracic artery stenosis, coronary artery bypass grafting, left anterior descending artery stenosis, 320-detector row computed tomography, adenosine thallium-201 myocardial perfusion scintigraphy

Department of Cardiovascular Surgery, Fujita Health University, Dengakugakubo, Kutukake-cho, Toyoake, Aichi, Japan

Received: February 21, 2011; Accepted: May 24, 2011

Corresponding author: Yasushi Takagi, MD. Department of Cardiovascular Surgery, Fujita Health University, 1-98 Dengakugakubo, Kutukake-cho, Toyoake, Aichi 470-1192, Japan Email: ytakagi@fujita-hu.ac.jp

(C)2011 The Editorial Committee of Annals of Thoracic and Cardiovascular Surgery. All rights reserved.

\section{Introduction}

Although invasive coronary angiography (ICA) is a standard method for evaluating coronary artery bypass grafts, 64-slice multidetector computed tomography (64MDCT) has become more popular because of its less invasive nature. ${ }^{1,2)}$ However, 64-MDCT has certain limitations despite recent improvements. ${ }^{3-6)}$ Recently, we used 320-detector row computed tomography (area-detector computed tomography; 320-ADCT) in order to lower 


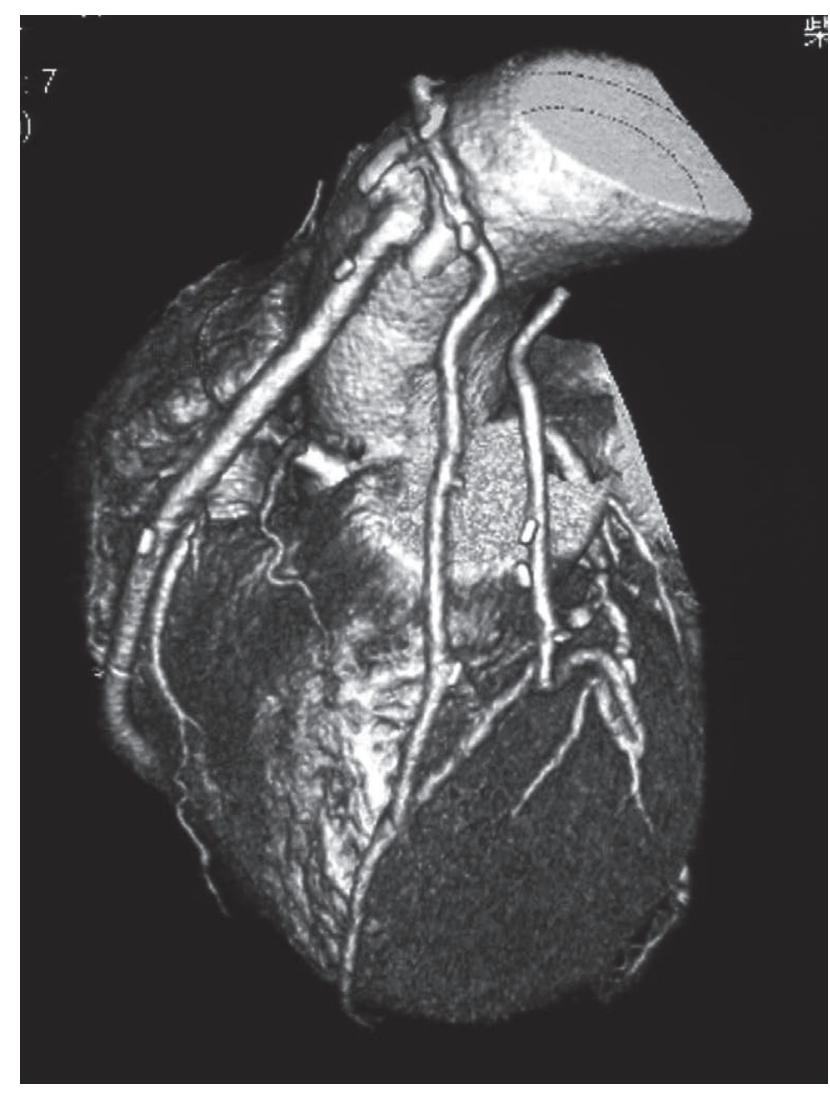

Fig. 1 A 67-year-old male who underwent coronary artery bypass grafting with the right internal thoracic artery to the left anterior descending artery, the left internal thoracic artery to the diagonal branch, and the saphenous vein to the posterior descending artery. Three-dimensional volume rendering image with 320-detector row computed tomography (320-ADCT) demonstrates excellent image quality.

contrast and radiation use, with excellent results (Fig. 1). ${ }^{7,8)}$

The use of an in situ internal thoracic artery (ITA) for replacing the left anterior descending artery (LAD) has been widely accepted as a standard coronary artery bypass grafting $(\mathrm{CABG})$ strategy that provides long-term patency and improves late survival. ${ }^{9-12)}$ However, it has been suggested that mild or moderate stenosis in the LAD is associated with competitive flow, thereby leading to a decreased antegrade flow in the ITA graft, which may lead to graft failure. ${ }^{13-16)}$

The purpose of this study was to evaluate the influence of clinical parameters and the degree of LAD stenosis on postoperative ITA graft stenosis, as well as the relationship between postoperative regional ischemia of the LAD territory, as assessed by adenosine thallium-201 myocardial perfusion scintigraphy (Tl-201-MPS), and postoperative ITA graft stenosis using 320-ADCT.

\section{Materials and Methods}

We evaluated 101 patients who underwent CABG using ITA grafts between January 2008 and September 2010. ITA grafting to the LAD was indicated when stenosis in the left main trunk was $\geq 50 \%$ or that in the LAD was $\geq 75 \%$. On-pump CABG was indicated when it was difficult to maintain systolic blood pressure $\geq 100 \mathrm{mmHg}$ due to acute myocardial infarction, severe left main trunk stenosis, or ejection fraction $\leq 20 \%$. Otherwise, off-pump CABG was performed. Patients with creatinine levels higher than $1.5 \mathrm{mg} / \mathrm{dL}$ were excluded from this study. Included patients had undergone both 320-ADCT and adenosine Tl-201-MPS 2-3 months after CABG. All patients provided written informed consent after the methods and extra cost of the investigations had been fully explained.

Conventional ICA was performed according to standard techniques within 2 months before CABG. The angiograms were analyzed by 2 interventional cardiologists. Lumen diameter reduction in the left main trunk and LAD was quantified in 2 orthogonal views.

All ITA graft studies were performed using the 320ADCT scanner (Aquilion ONE; Toshiba Medical Systems, Tokyo, Japan). The parameters were as follows: detector configuration, $320 \times 0.5 \mathrm{~mm}$; gantry rotation time, 200-580 s/rotation depending on R-R time; tube potential, $12 \mathrm{kV}$; and tube current, $0.35-0.4 \mathrm{~mA}$ depending on body habitus. Patients were imaged with prospective electrocardiogram (ECG) gating. The fraction of the R-R interval during which the patient was exposed was 60$80 \%$ for patients with heart rates $<67$ beats/min and $35-$ $80 \%$ for patients with heart rates $\geq 67$ beats $/ \mathrm{min}$. Regardless of rhythm, when images were acquired at heart rates $<67$ beats/min, scanning was completed within a single $\mathrm{R}-\mathrm{R}$ interval using a $180^{\circ}$ segment. A switch to multisegment acquisition was automatically triggered when the heart rate was $\geq 67$ beats/min, resulting in image acquisition over 2-3 R-R intervals. ${ }^{8)}$ A bolus of $1.1 \mathrm{~mL} / \mathrm{kg}$ iopromide was injected over $18 \mathrm{~s}$, followed by a $20-\mathrm{mL}$ saline chaser at a flow rate of $3 \mathrm{~mL} / \mathrm{s}$. Bolus tracking in the descending aorta was performed using a 200 Hounsfield unit threshold. Isosorbide dinitrate spray $1.25 \mathrm{mg}$ (Nitorol Spray; Isosorbide dinitrate, Eisai, Tokyo, Japan) was administered. No patients received additional beta-blockers before imaging. Image data from the CT were transferred to a workstation (ZIO STATION System 1000; Ziosoft, Tokyo, Japan) equipped with coronary artery analysis software. Image interpretation was based on volume 
Table 1 Patient characteristics and results of 320-ADCT and adenosine Tl-201-MPS

\begin{tabular}{lc}
\hline Number of Patients & 101 \\
Age (years) & $68.1 \pm 1.4$ \\
Female & $15(15 \%)$ \\
Off-pump CABG & $87(86 \%)$ \\
Redo surgery & $3(3 \%)$ \\
Distal anastomosis & $3.3 \pm 0.7$ \\
ITA graft anastomosed to the LAD & $92(91 \%)$ \\
$\quad$ Left ITA & $9(9 \%)$ \\
$\quad$ Right ITA & $71(70 \%)$ \\
Stenosis $\leq 75 \%$ of the LAD with preoperative ICA & $9(9 \%)$ \\
Stenosis $>50 \%$ of the ITA anastomosed to the LAD with 320-ADCT & $23(23 \%)$ \\
Regional ischemia of the LAD territory with adenosine Tl-201-MPS & \\
\hline 320-ADCT: $320-$ detector row computed tomography (area-detector computed tomography); Tl-201- \\
MPS: thallium-201 myocardial perfusion scintigraphy; CABG: coronary artery bypass grafting; ITA: \\
internal thoracic artery; LAD: left anterior descending artery; ICA: invasive coronary angiography
\end{tabular}

rendering images, multiplanar reconstruction images, curved planar reconstruction images, cross-sectional images, and maximum-intensity projection images. Images were analyzed independently by 2 experienced radiologists who were unaware of the clinical data and were blinded to the preoperative ICA results. ITA graft stenosis was defined as a reduction in the luminal diameter by $\geq 50 \%$.

Adenosine Tl-201-MPS was performed according to the American College of Cardiology/American Heart Association/American Society of Nuclear Cardiology guidelines for the clinical use of cardiac radionuclide imaging. ${ }^{17)}$ For each patient, stress was induced pharmacologically through intravenous infusion of adenosine (6-min infusion of $140 \mu \mathrm{g} / \mathrm{kg} / \mathrm{min}$ ), as described by Nishimura et al. ${ }^{18)}$ The patient's standard ECG, vital signs, and general condition were continuously monitored during the stress protocol. At 3 min after the continuous infusion of adenosine, $111 \mathrm{MBq}$ thallium-201 (FUJI FILM RI Pharma Co., Ltd., Tokyo, Japan) was injected intravenously and flushed with saline. Early single photon emission tomography (SPECT) was performed $10 \mathrm{~min}$ after the adenosine stress test; late SPECT was performed $4 \mathrm{~h}$ thereafter. SPECT images were acquired using a dual-headed SPECT gamma camera (ADAC VERTEX-plus; EPIC, USA). Tomographic reconstruction was performed using a standard filtered back-projection technique with a ramp filter to produce a transaxial tomogram. No scatter or attenuation correction was applied. From these transaxial tomograms, the long axis of the left ventricle was identified, and oblique-angled tomograms were generated (i.e., vertical long-axis, short-axis, and horizontal long-axis tomograms). Four experienced radiologists and cardiolo- gists visually and independently analyzed the SPECT images. The slices were displayed sequentially to assess myocardial perfusion in each vascular territory. ${ }^{19)}$ The presence or absence of redistribution related to the early images was visually judged in the 4-h images, which were read as positive or negative for ischemia.

Patients were divided into 2 groups. Group A included patients without ITA graft stenosis, whereas Group B included patients with ITA graft stenosis. We compared the clinical parameters, degree of LAD stenosis, and regional myocardial ischemia of the LAD territory between the 2 groups.

Statistical analysis was performed using StatView, version 5.0 (Abacus Concepts, Berkeley, CA). Quantitative variables that approximated a normal distribution are presented as mean \pm standard deviation. Continuous variables were analyzed using unpaired $t$ tests. Categorical variables were analyzed using Fisher's exact tests. $\mathrm{P}<0.05$ was considered to be statistically significant.

\section{Results}

Patient profiles are shown in Table 1. The mean age of the 101 patients was $68.1 \pm 1.4$ years. There were 15 women (15\%) among our subjects. Off-pump CABG was performed in 87 patients (86\%); of these, redo CABG was performed in 3 patients. The mean number of distal anastomoses was $3.3 \pm 0.7$. The left ITA was anastomosed to the LAD in 92 patients (91\%), whereas the right ITA was used in 9 patients $(9 \%)$. Stenosis of the LAD, including the left main trunk, on preoperative ICA was $\leq 75 \%$ in 30 patients (30\%). Significant ITA stenosis was noted in 9 patients (30\%) using postoperative 320-ADCT (Fig. 2). 

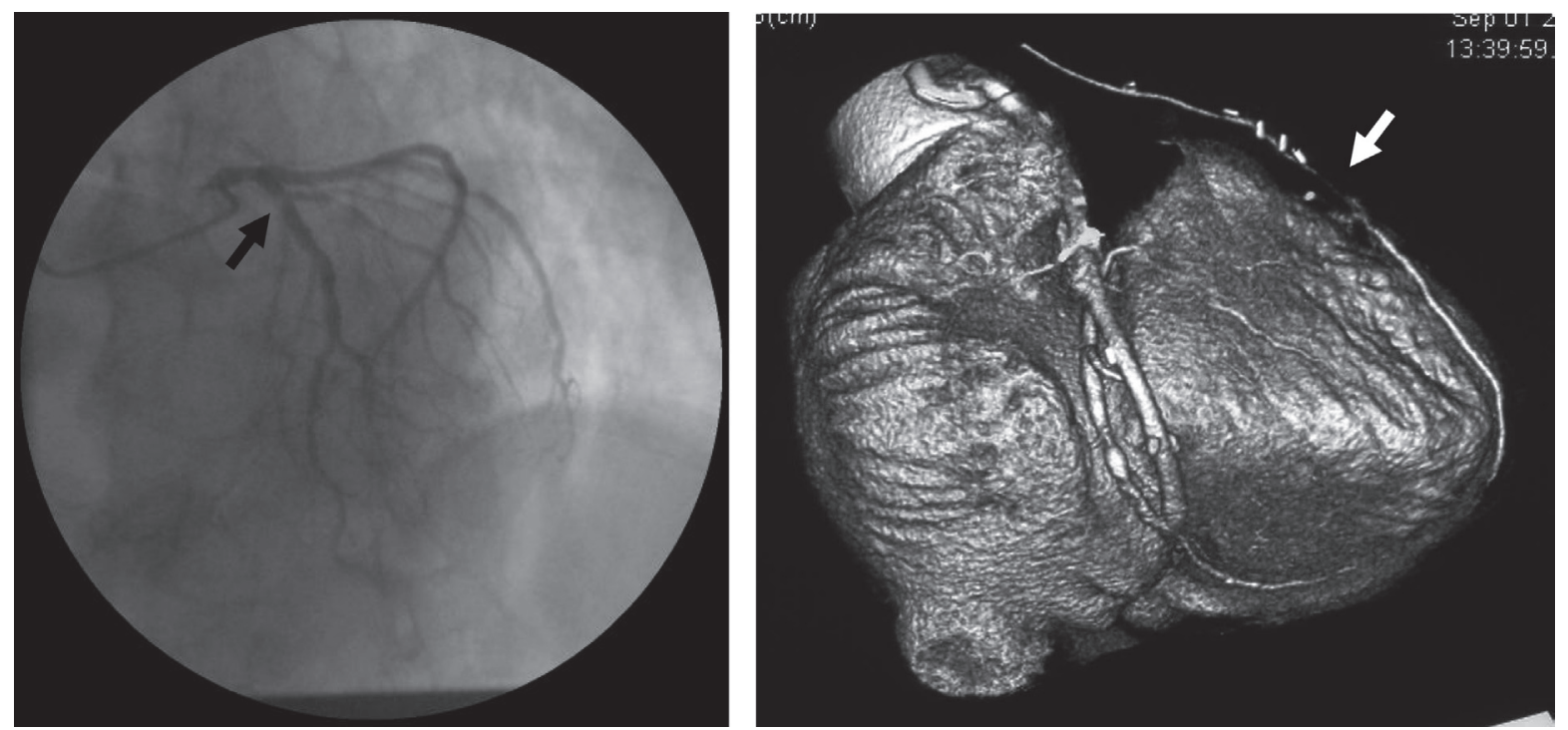

Fig. 2 An 80-year-old male who underwent coronary artery bypass grafting with the left internal thoracic artery to the left anterior descending artery and the saphenous vein to the right coronary artery (RCA). (A) Preoperative invasive coronary angiography (ICA) shows 75\% stenosis of the left anterior descending artery (black arrow). (B) Three-dimensional volume rendering image with 320-ADCT demonstrates that the internal thoracic artery has a string sign proximal to the distal anastomosing site (white arrow).

Table 2 Comparison of clinical characteristics and results of adenosine Tl-201-MPS between patients with and without ITA stenosis

\begin{tabular}{|c|c|c|c|}
\hline & $\begin{array}{c}\text { Group A } \\
(\mathrm{n}=92)\end{array}$ & $\begin{array}{c}\text { Group B } \\
(n=9)\end{array}$ & P-value \\
\hline Age (years) & $68 \pm 7.0$ & $68 \pm 7.7$ & 0.9170 \\
\hline Female & $14(15 \%)$ & $1(11 \%)$ & $>0.9999$ \\
\hline Off-pump CABG & $12(13 \%)$ & $3(33 \%)$ & 0.1285 \\
\hline Distal anastomosis & $3.3 \pm 1.1$ & $3.1 \pm 1.3$ & 0.5213 \\
\hline Right ITA anastomosed to the LAD & $8(7 \%)$ & $1(11 \%)$ & 0.5804 \\
\hline Stenosis $\leq 75 \%$ of the LAD with preoperative ICA & $22(24 \%)$ & $8(89 \%)$ & 0.0002 \\
\hline Regional ischemia of LAD territory with adenosine Tl-201-MPS & $23(25 \%)$ & $0(0 \%)$ & 0.1144 \\
\hline
\end{tabular}

Tl-201-MPS: thallium-201 myocardial perfusion scintigraphy; ITA: internal thoracic artery; CABG: coronary artery bypass grafting; LAD: left anterior descending artery; ICA: invasive coronary angiography

Group A: patients without ITA stenosis; Group B: patients with ITA stenosis

Three patients with ITA stenosis underwent ICA after 320-ADCT, which showed significant stenosis (string sign) and competitive flow in the same portion. Adenosine Tl-201-MPS demonstrated regional ischemia of the LAD territory in 23 patients (23\%) who had no ITA stenosis.

There were no differences between Groups A and B with respect to age, sex, off-pump CABG, the number of distal anastomoses, right/left ITA anastomosed to the LAD, or regional ischemia of the LAD territory. Eight patients (89\%) in Group B and 22 patients (24\%) in Group A had $\leq 75 \%$ LAD stenosis. A significant differ- ence in this parameter was thus seen between the 2 groups. No patients in Group B were found to have regional myocardial ischemia of the LAD territory according to adenosine Tl-201-MPS results (Table 2).

\section{Discussion}

Although ICA has long been the gold standard method for evaluating bypass grafts, it entails life-threatening risks such as arrhythmia, stroke, coronary artery dissection, peripheral artery occlusion, and bleeding..$^{19,20)}$ Recently, 64-MDCT has become more popular, because it is 
less invasive and can be performed on an outpatient basis. Furthermore, recent reports have demonstrated the benefits of 64-MDCT as a modality for evaluating bypass graft patency with its high sensitivity and high negative predictive value, proving that MDCT is a reliable method for detecting non-diseased grafts without significant stenosis. ${ }^{6,21)}$ Despite these benefits, the evaluation of patients using 64-MDCT has certain limitations. It is difficult to diagnose patients who have a rapid heart rate or arrhythmia, because of motion artifacts due to a relatively low temporal resolution. ${ }^{21-23)}$ High radiation exposure comprises another concern for the use of 64-MDCT.

In contrast, the latest-generation 320-ADCT system represents a significant advance from 64-MDCT technology. It enables $16-\mathrm{cm}$ craniocaudal coverage in a rotation time of $350 \mathrm{~ms}$, imaging the entire coronary tree in a single gantry rotation within a single heartbeat. These technical improvements allow axial volumetric imaging with single-beat image acquisition, uniform contrast enhancement, and an ability to study patients in atrial fibrillation with excellent image quality. ${ }^{8}$ Further, this technique is expected to require lower contrast and radiation doses while maintaining excellent image quality as compared to $64-\mathrm{MDCT}^{7,24)}$

The use of an in situ ITA graft for repairing the LAD is widely accepted as a gold standard strategy that provides long-term patency and improves late survival. The patency rate has been shown to range from $85 \%$ to $95 \%$ at 7-10 years. ${ }^{9-11)}$ However, occlusion of the ITA graft significantly limits future revascularization options. Doppler studies have suggested that moderate stenosis in the target vessel is associated with competitive flow, thereby leading to decreased antegrade flow in the arterial graft, which may lead to early failure (string sign). ${ }^{13-15)}$ Recently, Berger et al. reported that the degree of stenosis in the native vessel is a major predictor of ITA graft occlusion. ${ }^{17}$ The ITA was anastomosed to the LAD in $96 \%$ of our recent $\mathrm{CABG}$ cases. In patients who underwent early postoperative assessment, ITA occlusion was less than $1 \%$, and, in the present study, no occluded ITAs were observed. However, 9 patients (9\%) had significant stenosis of the ITA anastomosed to the LAD. Among these patients, $8(89 \%)$ had $\leq 75 \%$ native vessel stenosis. In agreement with previous reports, this finding indicates that patients with significant ITA stenosis have significantly higher rates of $\leq 75 \%$ native vessel stenosis than those with non-significant ITA stenosis. In all these cases, ITA stenosis existed just proximal to the anastomosing site. Although the length of the stenoses was variable, all stenoses were found to occur concentrically. The postoperative ICA of patients with ITA stenosis showed a string sign with competitive flow in the same portion. Although 320-ADCT can clearly detect whether the graft is stenotic or not, it cannot demonstrate the flow direction in the graft. On the other hand, artifacts from the metallic clip and calcification prevent clear visualization during 320ADCT, interfering with accurate diagnosis of the condition of the bypass grafts and the native coronary artery. ${ }^{4-6)}$ To obtain clear visualization, we have attempted to avoid using the metallic clip at our institution, especially at anastomotic sites. Although it was difficult to diagnose flow competition in the 9 patients with significant ITA graft stenosis, based on recent reports, we considered that the diagnostic accuracy of 320-ADCT was equal to or better than that of 64-MDCT., ${ }^{7,8)}$ In order to detect flow competition or slow flow in the ITA, a dynamic study of the ITA graft can be performed using isophasic 320ADCT images, although this would require increased contrast and radiation doses. Further development of these techniques and protocols is required for the obtainment of such 320-ADCT images while maintaining low contrast and radiation doses.

Perfusion defects with exercise Tl-201-MPS have been shown to predict death and non-fatal myocardial infarctions in patients who have undergone $\mathrm{CABG}^{25,26)} \mathrm{How}-$ ever, exercise testing is often inadequate and has a negative impact on the sensitivity of scintigraphy for detecting graft disease, because a substantial number of patients referred for imaging cannot exercise or attain maximal heart rates, and many patients continue to use anti-ischemic medications after surgery. ${ }^{27-29)}$ In this study, we performed pharmacologic imaging, which may be advantageous in such patients. No patients with ITA stenosis had regional myocardial ischemia of the LAD territory, as evaluated by adenosine Tl-201-MPS, suggesting that there was no need for re-intervention despite LAD and ITA stenosis. On the other hand, 23 patients (23\%) without ITA stenosis had regional myocardial ischemia of the LAD territory. This finding may suggest the existence of non-bypassed native disease proximal or distal to the graft or insufficient flow in the ITA, which is expected to improve with the growth of the ITA graft. ${ }^{30)}$ Furthermore, false positive results in such scanning may be caused by previous myocardial infarctions. ${ }^{31)}$ In our study, none of the 101 patients showed any symptoms during followup or required re-intervention. Patients with creatinine levels higher than $1.5 \mathrm{mg} / \mathrm{dL}$ were evaluated only with postoperative adenosine Tl-201-MPS. ICA was performed 
when they were symptomatic or had a relatively large territory of myocardial ischemia.

In the present study, off-pump CABG was performed in $86 \%$ of patients, which is a greater frequency than that reported previously. Recent studies have shown that early outcomes of off-pump CABG are better than those of onpump CABG ${ }^{32-33)}$ However, it remains controversial whether late outcomes associated with off-pump CABG are better or worse than those associated with on-pump CABG. It is, therefore, important to follow up patients to compare the long-term graft patency between these 2 methods using non-invasive 320-ADCT and adenosine Tl-201-MPS.

\section{Conclusion}

Although our study was limited by the small number of cases remaining after exclusion of patients with renal failure, it showed that $\leq 75 \%$ LAD stenosis had a significant influence on ITA stenosis, without associated regional myocardial ischemia of the LAD territory. As previous reports have demonstrated that about $10 \%$ of ITA grafts occlude within 10 years after CABG mainly due to flow competition, it is important to follow up patients using non-invasive methods. We have shown here that ITA evaluation with 320-ADCT and adenosine Tl-201MPS may be useful for the long-term follow-up of patients after $\mathrm{CABG}$.

\section{References}

1) Budoff MJ, Achenbach S, Blumenthal RS, et al. Assessment of coronary artery disease by cardiac computed tomography: a scientific statement from the American Heart Association Committee on Cardiovascular Imaging and Intervention, Council on Cardiovascular Radiology and Intervention, and Committee on Cardiac Imaging, Council on Clinical Cardiology. Circulation 2006; 114: 1761-91.

2) Abbara S, Arbab-Zadeh A, Callister TQ, et al. SCCT guidelines for performance of coronary computed tomographic angiography: a report of the Society of Cardiovascular Computed Tomography Guidelines Committee. J Cardiovasc Comput Tomogr 2009; 3: 190-204. Epub 2009 Mar 31.

3) Yoo KJ, Choi D, Choi BW, et al. The comparison of the graft patency after coronary artery bypass grafting using coronary angiography and multi-slice computed tomography. Eur J Cardiothorac Surg 2003; 24: 86-91; discussion 91.

4) Yamamoto M, Kimura F, Niinami H, et al. Noninva- sive assessment of off-pump coronary artery bypass surgery by 16-channel multidetector-row computed tomography. Ann Thorac Surg 2006; 81: 820-7 .

5) Andreini D, Pontone G, Ballerini G, et al. Bypass graft and native postanastomotic coronary artery patency: assessment with computed tomography. Ann Thorac Surg 2007; 83: 1672-8.

6) Tochii M, Takagi Y, Anno H, et al. Accuracy of 64slice multidetector computed tomography for diseased coronary artery graft detection. Ann Thorac Surg 2010; 89: 1906-11.

7) Rybicki FJ, Otero HJ, Steigner ML, et al. Initial evaluation of coronary images from 320-detector row computed tomography. Int J Cardiovasc Imaging 2008; 24: 535-46.

8) Nasis A, Leung MC, Antonis PR, et al. Diagnostic accuracy of noninvasive coronary angiography with 320-detector row computed tomography. Am J Cardiol 2010; 106: 1429-35.

9) Cameron A, Davis KB, Green G, et al. Coronary bypass surgery with internal-thoracic-artery grafts--effects on survival over a 15-year period. N Engl J Med 1996; 334: 216-9.

10) Cameron A, Davis KB, Green G, et al. Coronary bypass surgery with internal-thoracic-artery grafts--effects on survival over a 15-year period. N Engl J Med 1996; 334: 216-9.

11) Lytle BW, Blackstone EH, Loop FD, et al. Two internal thoracic artery grafts are better than one. J Thorac Cardiovasc Surg 1999; 117: 855-72.

12) Nasu M, Akasaka T, Okazaki T, et al. Postoperative flow characteristics of left internal thoracic artery grafts. Ann Thorac Surg 1995; 59: 154-61; discussion 161-2.

13) Hashimoto H, Isshiki T, Ikari Y, et al. Effects of competitive blood flow on arterial graft patency and diameter. Medium-term postoperative follow-up. J Thorac Cardiovasc Surg 1996; 111: 399-407.

14) Shimizu T, Hirayama T, Suesada H, et al. Effect of flow competition on internal thoracic artery graft: postoperative velocimetric and angiographic study. J Thorac Cardiovasc Surg 2000; 120: 459-65.

15) Berger A, MacCarthy PA, Siebert U, et al. Long-term patency of internal mammary artery bypass grafts: relationship with preoperative severity of the native coronary artery stenosis. Circulation 2004; 110; II-36-II-40.

16) Klocke FJ, Baird MG, Lorell BH, et al. ACC/AHA/ ASNC guidelines for the clinical use of cardiac radionuclide imaging--executive summary: a report of the American College of Cardiology/American Heart Association Task Force on Practice Guidelines (ACC/ AHA/ASNC Committee to Revise the 1995 Guidelines for the Clinical Use of Cardiac Radionuclide Imaging). Circulation 2003; 108: 1404-18.

17) Nishimura $S$, Mahmarian JJ, Boyce TM, et al. Quantitative thallium-201 single-photon emission computed tomography during maximal pharmacologic coronary vasodilation with adenosine for assessing coronary ar- 
tery disease. J Am Coll Cardiol 1991; 18: 736-45.

18) Cerqueira MD, Weissman NJ, Dilsizian V, et al. Standardized myocardial segmentation and nomenclature for tomographic imaging of the heart: a statement for healthcare professionals from the Cardiac Imaging Committee of the Council on Clinical Cardiology of the American Heart Association. J Nucl Cardiol 2002; 9: $240-5$.

19) Krone RJ, Johnson L, Noto T. Five year trends in cardiac catheterization: a report from the Registry of the Society for Cardiac Angiography and Interventions. Cathet Cardiovasc Diagn 1996; 39: 31-5.

20) Ammann P, Brunner-La Rocca HP, Angehrn W, et al. Procedural complications following diagnostic coronary angiography are related to the operator's experience and the catheter size. Catheter Cardiovasc Interv 2003; 59: 13-8.

21) Hoffmann U, Ferencik M, Cury RC, et al. Coronary CT angiography. J Nucl Med 2006; 47: 797-806.

22) Raff GL, Gallagher MJ, O'Neill WW, et al. Diagnostic accuracy of noninvasive coronary angiography using 64-slice spiral computed tomography. J Am Coll Cardiol 2005; 46: 552-7.

23) Delhaye D, Remy-Jardin M, Salem R, et al. Coronary imaging quality in routine ECG-gated multidetector CT examinations of the entire thorax: preliminary experience with a 64-slice CT system in 133 patients. Eur Radiol 2007; 17: 902-10.

24) Pasricha SS, Nandurkar D, Seneviratne SK, et al. Image quality of coronary 320-MDCT in patients with atrial fibrillation: initial experience. AJR Am J Roentgenol 2009; 193: 1514-21.
25)Lauer MS, Lytle B, Pashkow F, et al. Prediction of death and myocardial infarction by screening with exercise-thallium testing after coronary-artery-bypass grafting. Lancet 1998; 351: 615-22.

26) Miller TD, Christian TF, Hodge DO, et al. Prognostic value of exercise thallium-201 imaging performed within 2 years of coronary artery bypass graft surgery. J Am Coll Cardiol 1998; 31: 848-54.

27) Verani MS, Mahmarian JJ. Myocardial perfusion scintigraphy during maximal coronary artery vasodilation with adenosine. Am J Cardiol 1991; 67: 12D-17D.

28) Iskandrian AS, Heo J, Kong B, et al. Effect of exercise level on the ability of thallium-201 tomographic imaging in detecting coronary artery disease: analysis of 461 patients. J Am Coll Cardiol 1989; 14: 1477-86.

29) Mahmarian JJ, Verani MS. Exercise thallium-201 perfusion scintigraphy in the assessment of coronary artery disease. Am J Cardiol 1991; 67: 2D-11D.

30) Nakayama Y, Sakata R, Ura M. Growth potential of left internal thoracic artery grafts: analysis of angiographic findings. Ann Thorac Surg 2001; 71: 142-7.

31) Khoury AF, Rivera JM, Mahmarian JJ, et al. Adenosine thallium-201 tomography in evaluation of graft patency late after coronary artery bypass graft surgery. J Am Coll Cardiol 1997; 29: 1290-5.

32) Reston JT, Tregear SJ, Turkelson CM. Meta-analysis of short-term and mid-term outcomes following off-pump coronary artery bypass grafting. Ann Thorac Surg 2003; 76: 1510-5.

33) Puskas JD, Kilgo PD, Lattouf OM, et al. Off-pump coronary bypass provides reduced mortality and morbidity and equivalent 10-year survival. Ann Thorac Surg 2008; 86: 1139-46; discussion 1146. 